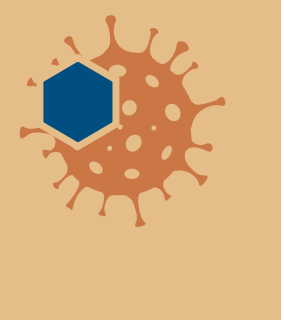

\title{
Government funding ramps up COVID-19 research around the globe
}

$\mathrm{M}$ aterials research laboratories in the United States have received urgent funding for research on SARS-CoV-2, the novel coronavirus that causes COVID19. However, their call to the US federal government for a relief package to cover research projects that have been put on hold due to the pandemic remains unheeded.

On March 27, the Coronavirus Aid, Relief, and Economic Security (CARES) Act was signed into law. The over USD \$2 trillion economic relief package includes assistance for operating costs of government laboratories and programs as well as new funding for $R \& D$ against the 2019 novel coronavirus or COVID-19. For example, USD\$50 million goes to the Manufacturing Extension Partnership (MEP) to help small- and mid-size businesses recover from the economic effects of the coronavirus. It also covers operating costs for the national laboratories scientific user facilities under the US Department of Energy (DOE) to the tune of USD\$99.5 million.

For work related to solving the coronavirus pandemic, an additional USD \$28 million goes to DOE to support remote access by the department's employees enabling researchers, for example, to use its supercomputers through the COVID19 HPC (High Performance Computing) Consortium (covid19-hpc-consortium. org). The consortium reported over 40 active projects, as of mid-May. Along with the user facilities, members of the consortium include IBM, AMD, and Intel from industry; the Massachusetts Institute of Technology and Rensselaer Polytechnic Institute from academia; and numerous

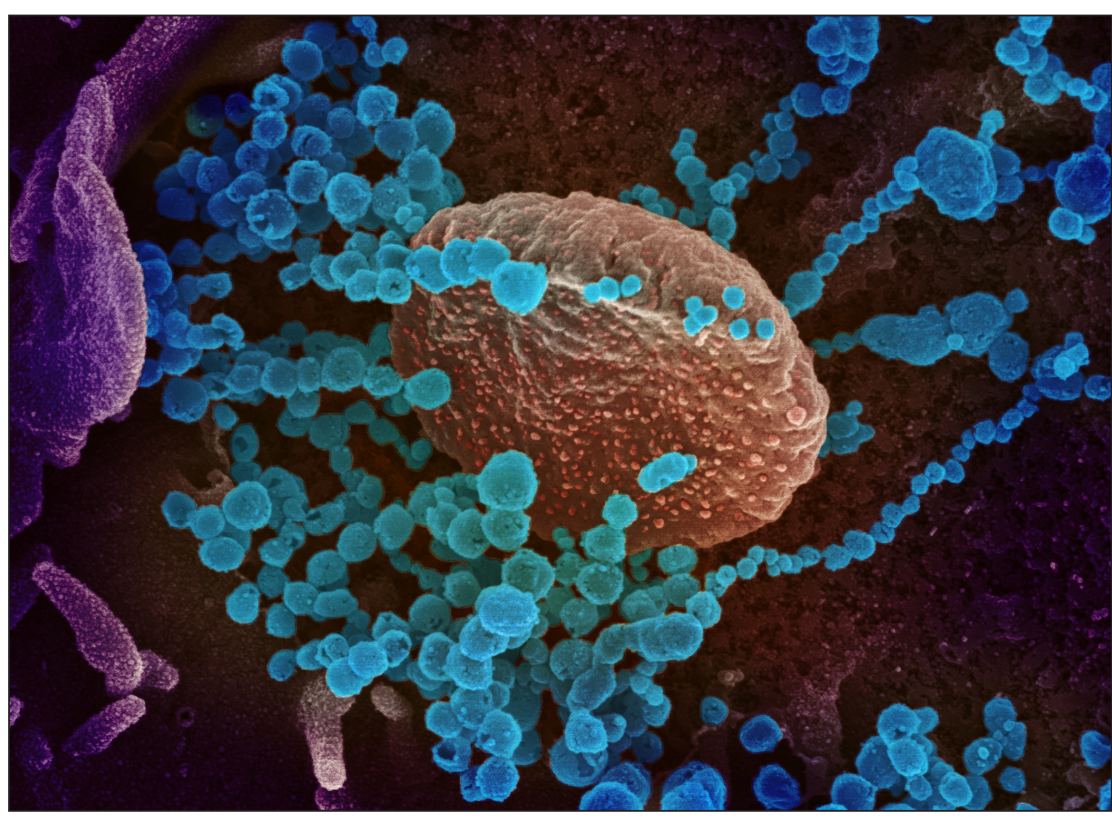

This scanning electron microscope image shows SARS-CoV-2 (round blue objects) emerging from the surface of cells cultured in the laboratory. SARS-CoV-2, also known as 2019-nCoV, is the virus that causes COVID-19. The virus shown was isolated from a patient in the United States. Credit: NIAID-RML. supercomputer centers supported by the National Science Foundation (NSF). Another USD\$28 million goes to support DOE employees working remotely for other COVID-19-related work.

The National Institute of Standards and Technology (NIST) receives USD\$6 million to continue operations, including research and measurements to improve on COVID-19 testing and diagnostics.

The CARES Act also provides funding for new R\&D to combat COVID19. NSF is spending USD $\$ 75$ million in its Rapid Response Research (RAPID) Grants program toward nonmedical R\&D to better understand the coronavirus. Proposals may be up to USD $\$ 200,000$ for one-year studies. Linda S. Sapochak, who is the Director of the Division of Materials Research (DMR), told MRS Bulletin that as of the beginning of June, "So far, NSF has invested in 699 awards specifically focused on COVID-19, totaling USD\$96,748,372. Of those projects, NSF's Division of Materials Research has provided funding or co-funding for 18 RAPID/EAGER awards that capitalize on or advance materials research, and we have some more in the pipeline, actually."

Sapochak points to grants from her division going to materials researchers across the various programs at DMR. For example, she says, David Grier at New York University is awarded a grant from the DMR Condensed Matter Physics program to leverage fast, inexpensive optical techniques that detect the physical properties of viral pathogens, potentially speeding up testing. With a technique called holographic particle characterization, his group creates laser holograms of specially prepared test beads and uses those holograms to monitor small changes in the beads' properties that occur when molecules or virus particles bind to their surfaces, she says. 
Jiaxing Huang at Northwestern University received funding from the Solid State and Materials Chemistry program to develop a new self-sanitizing medical face mask that deactivates viruses on contact; Dominik Konkolewicz at Miami University in Ohio received support from the Polymers program to develop new synthetic polymer materials that disable and/ or contain lipid molecules from the virus so they can be used on or as high-contact surfaces to minimize virus transmission.

From the Biomaterials program, Jessica R. Kramer at The University of Utah received funding to identify forms of mucus that result in increased airborne COVID transmission and infection potentially to develop new therapeutics that disrupt the mucus binding associated with COVID-19, or to help better identify populations most vulnerable to its transmission and infection. Kramer's team, Sapochak says, will simulate cough droplets produced from different types of mucus and use human cells coated with varied mucus.

Paresh Ray, a professor of chemistry at Jackson State University in Mississippi has been awarded an NSF RAPID Grant for R\&D on an immediate diagnostic test

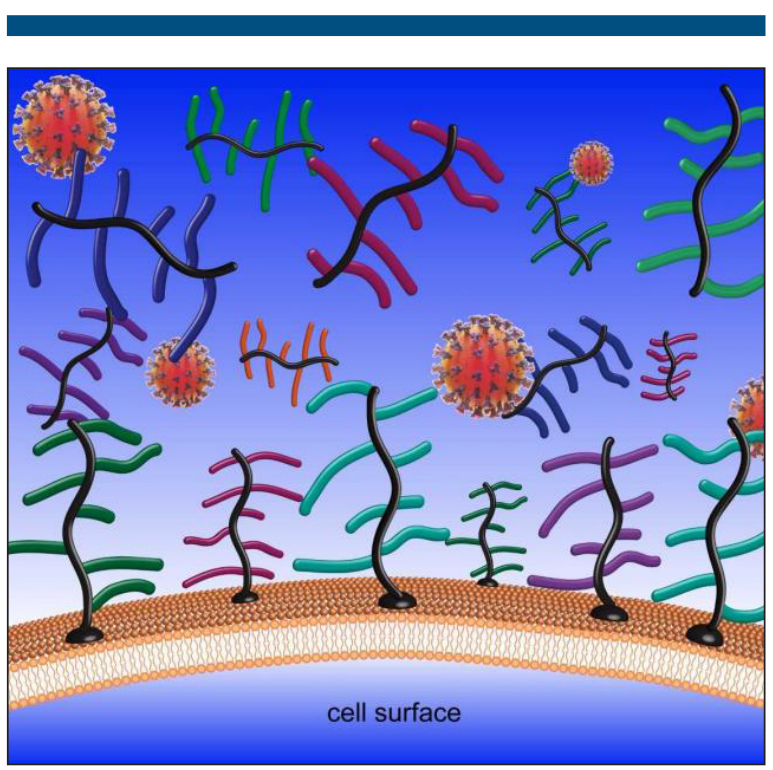

In this graphic, coronaviruses interact with mucus proteins at the human cell surface. Jessica R. Kramer (The University of Utah) is exploring how mucus plays a part in transferring coronaviruses from person to person. This research could help health officials better understand who would spread the virus more or how to develop effective quarantine measures for high-risk populations. Credit: Zachary S. Clauss and Jessica R. Kramer/The University of Utah College of Engineering. that relies on a nanomaterials-based technology. As an example of keeping within the broader impact value of NSF, Ray combines the special funding for COVID-19 research with funding from the NSF Partnerships for Research and Education in Materials (PREM) Program. According to Ray's proposal, “The RAPID program will serve as the bridge among scientific research, technological development, and educational activities for African American students."

Ray told MRS Bulletin that this grant supports research based on the outcome of his group's previous research that was accomplished through funding from the PREM program in which they developed a bio-conjugated nanostructure-based approach for specific identification of different pathogenic microorganisms such as Dengue virus, Zika virus, and West Nile virus, as well as Rotavirus and drug-resistant bacteria. Ray's goal now is to build from previous results to develop "sensitive, fast, and simple-touse optical techniques to detect viral-RNA using Raman spectroscopy, which has the capability for fingerprint identification of COVID-19." This will be accomplished, Ray says, by measuring the Raman signal from COVID-19 RNA.

In further distribution of funds, USD\$415 million is designated from the CARES Act to the US Department of Defense for the development of vaccines and treatment as well as for laboratory operations and purchasing of diagnostic tests.
For vaccine, therapeutic, and diagnostic R\&D related to COVID-19, the National Institutes of Health (NIH) has altogether received USD $\$ 1.78$ billion-USD $\$ 945.5$ million from the CARES Act and the remainder from a previously approved supplemental funding package.

In May, the Democratic staff of the US House Committee on Appropriations prepared the Heroes Act (H.R. 6800), a USD $\$ 3$ trillion supplemental appropriations act for coronavirus recovery. It passed a House vote on May 15 and includes additional provisions for COVID-19-related research.

Under the Heroes Act, NSF is to receive "USD\$125 million to prevent, prepare for, and respond to coronavirus," including funds for R\&D, and the NIH is to use USD\$3.5 billion for therapeutics and vaccines related to COVID-19 within the Biomedical Advanced Research and Development Authority, which is the office designated to counter pandemics. As of the time of publication, the Heroes Act has not been signed into law.

The United States is not alone in creating emergency R\&D funding packages. An emergency economic package against COVID-19 passed in Japan provides $¥ 1,809.7$ billion ( USD\$16 billion) to develop "preventive measures against the spread of infection and medical treatment structures, as well as pharmaceuticals.” Out of this funding, $¥ 20.0$ billion 
( USD\$186 million) goes to medical R\&D through industry-academia-government collaborations within Japan. Toward the development of vaccines, $¥ 10.0$ billion ( USD\$93 million) funds R\&D in Japan and $¥ 21.6$ billion ( USD\$201 million) toward work internationally.

In response to the pandemic, Singapore's Deputy Prime Minister and Minister of Finance Heng Swee Keat presented four budgets to Parliament between February and May. Due to previous financial planning, Singapore had built up a substantial reserve from which, by April, they withdrew up to S\$21 billion ( USD $\$ 15$ billion) to address the pandemic. Heng told Parliament, "[A]s a small country without any natural resources and highly dependent on imports, our reserves are vital to our overall economy and financial stability and our well-being. They provide a key defense for Singapore in terms of crisis."

For scientific research and innovation, Singapore also already has a plan in place, called the Research Innovation Enterprise 2020 Plan. From 2011 to 2015 , the plan was budgeted for $\mathrm{S} \$ 16$ billion ( USD $\$ 11.5$ billion), which was increased to S\$19 billion ( USD\$14 billion) in the next phase of 2016-2020. Two of the focus areas are "Advanced Manufacturing and Engineering" and "Health and Biomedical Sciences."

During his addresses to Parliament for the recent budget plans, Heng made note that Singapore's investment in scientific research had already helped in the fight against COVID-19. For example, the Agency for Science, Technology and Research known as A*STAR reported that "Fortitude Kit 2.0, a diagnostic kit developed by A*STAR and Tan Tock Seng Hospital, can accurately detect the presence of the SARS-CoV-2 virus, which causes COVID-19. It has been implemented in 13 Singapore hospitals and laboratories, public and private, and deployed to more than 20 countries globally."

Heng said, "We will continue to make steady investments in $\mathrm{R} \& \mathrm{D}$, under our masterplan for Research, Innovation and Enterprise. I will provide more details on this towards the end of the financial year." Specific for COVID-19-related research, the Singapore National Health Innovation

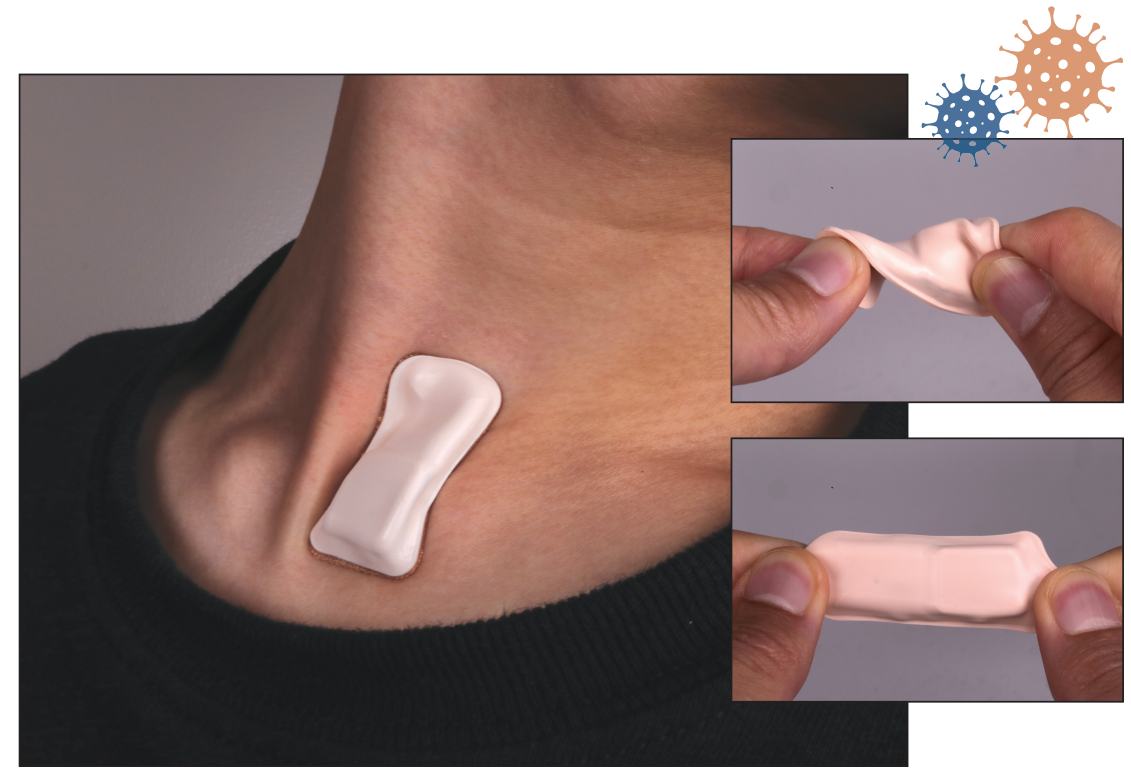

Soft, wireless electronic sensor mounted on the suprasternal notch enables continuous recordings of mechano-acoustic signatures of key symptoms of COVID-19-heart rate variability, respiration rate and respiratory sounds, cough (frequency, intensity), and temperature. Credit: John Rogers.

Centre issued a call for proposals in areas including devices, in vitro diagnostics, and therapeutics.

In April, the World Health Organization (WHO) hosted a virtual event putting out a call for action in the fight against the crippling disease. The EU responded with a pledge of $€ 1$ billion to Horizon 2020, the EU program for research and innovation. This funding adds to the $€ 352$ million that had already been mobilized to the program since January 2020. EU funding supports R\&D for tests, treatments, and vaccines as well as for disruptive innovations regarding COVID-19. Researchers wishing to travel abroad for collaboration will also find opportunities under the Marie Skłodowska-Curie Actions-Individual Fellowships for COVID-19-related work.

On April 20, the European COVID19 Data Platform was launched where researchers share data sets such as, for example, DNA sequences and protein structures.

The European Commission's Innovative Medicines Initiative (IMI), which originally received $€ 45$ million in response to the coronavirus outbreak, has had its total (public and private) funding increased to $€ 117$ million as of May. The latest projects, as of the time of publication, include five for diagnostic tests that are miniaturized or combined with wearable electronics so as to be administered anywhere, and three for R\&D in treatments.

According to Róisín Owens, university lecturer in chemical engineering and biotechnology at the University of Cambridge, due to Brexit where the UK is scheduled to finalize its withdrawal from the EU at the end of the year, the funding situation is more complicated. However, the UK, similar to other countries across Europe, made funding available for specific COVID-19 R\&D. In its call for proposals, for example, UK Research and Innovation (UKRI) is inviting researchers to repurpose their existing UKRI standard grants to address COVID-19 priority areas. Currently the UKRI has no specific budget for this call, but is "interested in funding research of any scale that can demonstrate it will deliver impact in the project length," according to the UKRI website. The projects can be up to 18 months duration.

In countering the coronavirus crisis in South Africa, the federal government is particularly concerned about vulnerability on two fronts. With the burden of HIV and tuberculosis (TB), a significant percentage of the population faces high risk if contracting the disease. Second, due to border closings and the unavailability of supplies that have been quickly routed around the world, South Africa needs to be self-reliant. 
In a press release, the Minister of Higher Education, Science and Innovation, Dr. Blade Nzimande, said, "Through the DSI [Department of Science and Innovation], we are engaging with the Department of Health, the Medical Research Council, and the South African Health Products Regulatory Authority with a view to mobilizing funding, reprioritizing research strategies, and creating an enabling ethical and regulatory framework to facilitate research on the COVID-19 virus."

In March, to control the spread of the disease, DSI redirected R4 million ( USD\$218,000) from some of its projects for research related to the novel coronavirus, as reported by the Department of Science and Technology in a news release. Since then, the DSI set aside R12 million ( USD\$650,000) and would redirect an additional R30 million ( USD\$1.6 million) for research interventions.

\section{Government support for COVID-19 materials research}

Materials researchers can contribute to solving the COVID-19 pandemic in a variety of ways, from surface sanitation and personal protective gear to detection, treatment, and vaccines.
In early April, NSF began accepting proposals for its RAPID Grants program, and by the end of April announced over 250 awards, some of which went to materials researchers. In the area of detection, Krystal Pollitt of Yale University received a grant for $\mathrm{R} \& \mathrm{D}$ on a wearable device that detects SARS-CoV-2 in the environment. As the disease may be spread through airborne aerosols, Pollitt aims to develop an inexpensive wristband in which the membrane made out of a poly(dimethylsiloxane) (PDMS)-based material serves as a sorbent bar for lipidenveloped viruses, such as SARS-CoV-2. With the wristband, individuals can monitor their potential risk of exposure to the virus in any given environment.

John Rogers, the Louis Simpson and Kimberly Querrey Professor of Materials Science and Engineering at Northwestern University, received a grant for another type of self-monitoring device in which a flexible wireless patch worn on the skin works together with mechano-acoustic sensors and machine learning algorithms for early diagnosis and monitoring of the virus. Rogers told MRS Bulletin that his research group works with a number of clinical collaborators across the medical complex in Chicago. "Some of those collaborators reached out to us for help,"

he says, "with specific requests for skininterfaced, wireless sensors with capabilities specifically aligned to key symptoms for the COVID-19 disease."

With previous funding from a research branch of the US Department of Health \& Human Services, Rogers's group developed a mechano-acoustic sensing device platform. Now with the NSF RAPID Grant, his team-together with Naresh Shanbhag's research group at the University of Illinois - is adding the pulse oximeter ( $\mathrm{SpO} 2)$ monitoring functionality to the device. "Most of the NSF program, however, is focused around advance data analytics approaches and machine learning techniques that we will be developing jointly with Prof. Shanbhag," Rogers says.

When it comes to personal protective equipment (PPE), materials researchers have particularly turned to R\&D in face masks. Shirley Meng, who holds the Zable Endowed Chair Professor in Energy Technologies and is Professor of NanoEngineering and Materials Science at the University of California, San Diego (UCSD), is well known for her work on sustainability and energy storage. With the onset of the pandemic, Meng's research group was asked by the UCSD health system to help investigate the efficiency of different kinds of face masks, many from suppliers that are not FDA-approved. The research team turned its attention to the reusabil-

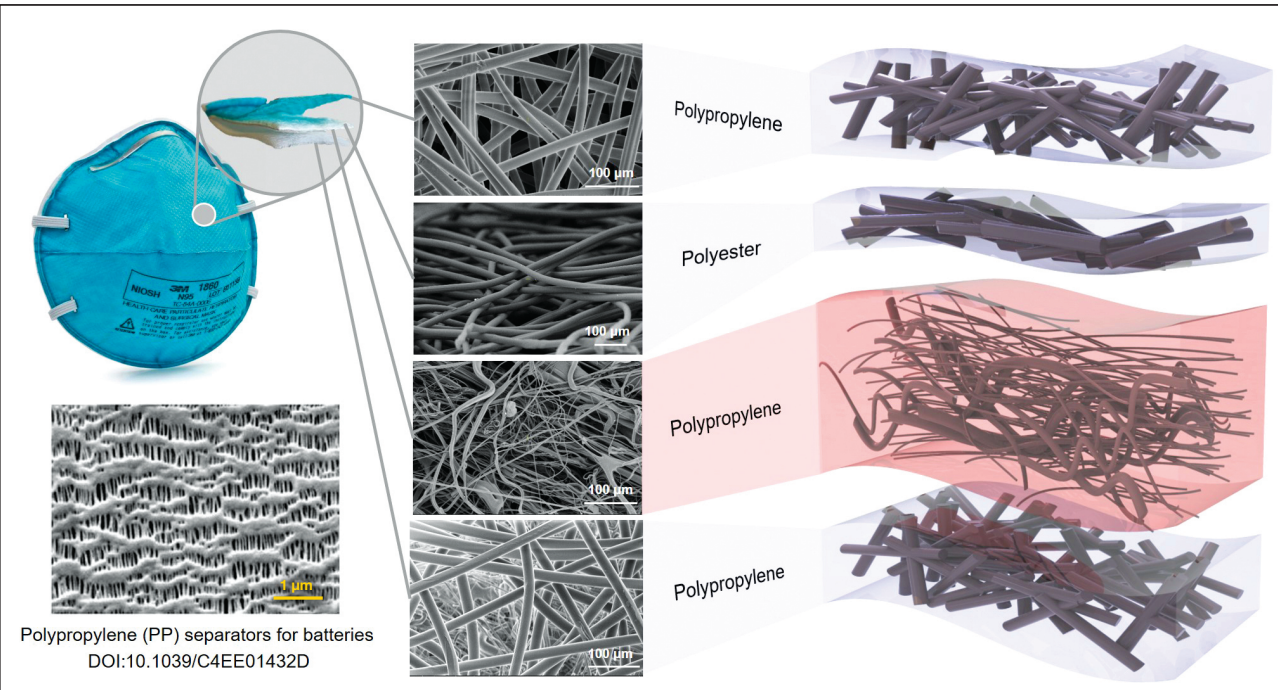

A 3M N95 mask model in comparison with a battery separator using a similar material. As polypropylene is utilized in both battery separators and masks, Shirley Meng and her research group at the University of California, San Diego, apply their tools for battery study on N95 masks as well. Credit: Shirley Meng, Min-cheol Kim, and Diyi Cheng. ity of N95 masks. Meng's group explored the question of what it takes to disinfect the mask without damaging it. Meng says that in their study they were surprised to learn that similar polymers are used in battery separator technology as the mask filter layers.

"Polyester and polypropylene are often used in battery separators for which we have studied using imaging tools and other physical properties measurement," Meng says. "We found that we can immediately deploy our tools to help fight COVID-19." 
Sterilization strategies under consideration include dry heat, steaming, hydrogen peroxide, and UV light. Meng's group determined that dry heat treatment at $70^{\circ} \mathrm{C}$ and $90^{\circ} \mathrm{C}$ is sufficient with one thermal cycle, but each repeated treatment after that deteriorates the mask. The research group also identified two areas in the design of the mask for improvement.

Mark Hersam, the Walter P. Murphy Professor of Materials Science and Engineering at Northwestern University, is also interested in the sterilization and reuse of N95 masks. At the early stages of the pandemic, his laboratory was contacted by Northwestern Memorial Hospital in Chicago about advice regarding decontamination protocols for N95 masks. Hersam told MRS Bulletin that when his group evaluated ultraviolet germicidal irradiation (UVGI), which is widely used for decontamination in hospitals, the tests revealed that the elastomers in the mask (e.g., head straps) irreversibly degrade with UVGI, which prevents the masks from fitting properly. This issue led his group to contemplate alternative elastomeric composite materials that would be resistant to UVGI, which is the basis of their NSF RAPID Grant.

With prior work Hersam's group has done with graphene oxide (GO), they are now investigating the possibility of using hydrated GO (hGO) to enhance sterilization as well as serve as a protective measure against the virus itself. "In particular," Hersam says, "we are exploring hydrated graphene oxide as an additive to elastomeric composites since hGO is known to absorb light strongly at ultraviolet wavelengths." And, based on their studies in the past few years of hGO in the context of antibacterial coatings, the researchers in Hersam's group are investigating it as an antiviral agent. Hydrated GO destroys lipid membranes of viruses. Hydrated GO, Hersam says, "has the advantage that it imparts antimicrobial activity to polymer surfaces, thus providing a secondary defense against virus contamination."

Having already experienced the SARS outbreak in 2003, researchers in Singapore stepped up efforts to tackle COVID-19. In January, Duke-NUS (National University of Singapore) Medical School announced

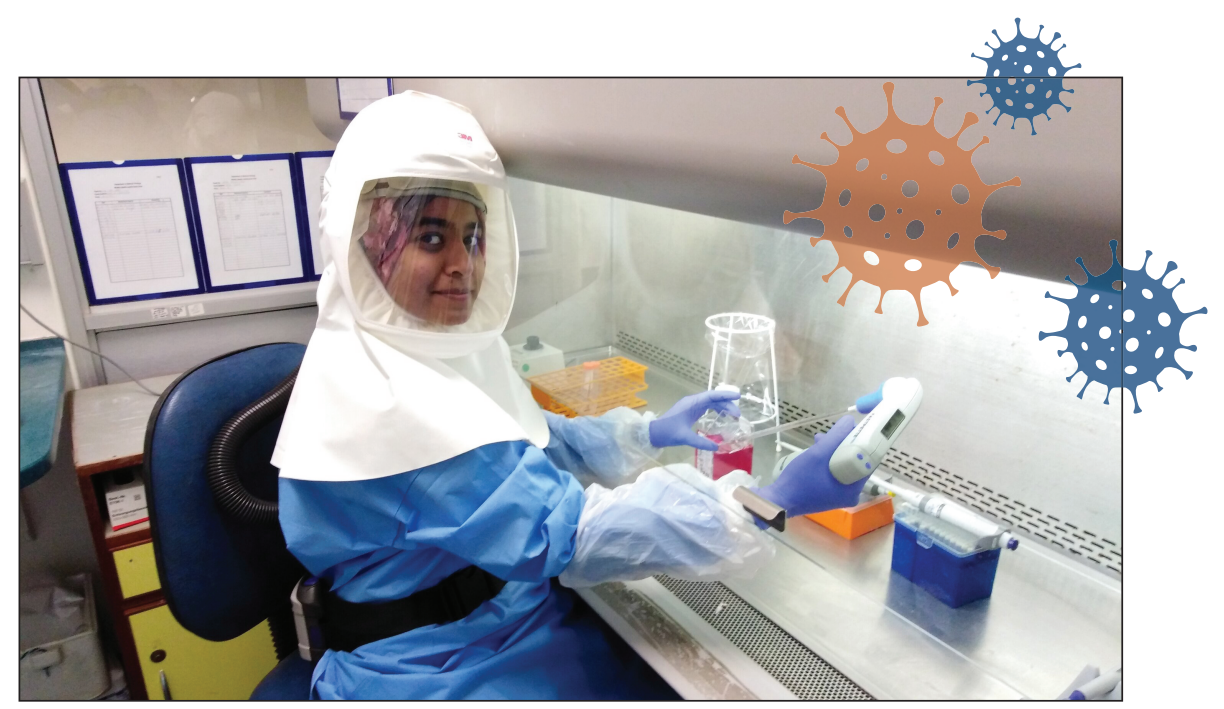

Tasnim Suliman of the University of the Western Cape (UWC) in South Africa working in a biosafety level-3 laboratory at Stellenbosch University's (SU) Division of Medical Virology where she cultures the SARS-CoV-2 virus for research and the evaluation of diagostic testing. Credit: UWC/SU.

that they have cultured the coronavirus. By February, on CNSNews in Singapore, Danielle Anderson, scientific director of the Duke-NUS ABSL3 facility, reported on two serological assays, one that uses parts of the virus' protein that is used in combination with the second assay, a virus neutralization test. At Nanyang Technological University, Peter Preiser, President's Chair in Biological Sciences, is developing a paper-based biomarker diagnostic test. For vaccine development, Duke-NUS is working with Arcturus Therapeutics STARR Technology platform that combines self-replicating RNA with a nanoparticle nonviral delivery system to produce proteins in the body.

In South Africa, according to the DSI, preliminary work on the development of vaccines started with the University of Cape Town, the Council for Scientific and Industrial Research, and Biovac Institute, which is a vaccine manufacturer in South Africa. Nzimande pointed to the need to locally manufacture reagents for testing kits because, before the border closings, they were being imported.

On April 1, the DSI reported, postdoctoral research fellow Tasnim Suliman of the University of the Western Cape obtained a laboratory isolate of SARSCoronavirus 2. In collaborative work with Stellenbosch University, Suliman has access to a biosafety level-3 (BSL-3) laboratory specifically designed for research on respiratory viruses. While most BSL-3 laboratories in the country are directed toward TB — caused by a bacterium-Suliman has succeeded in growing a BSL-3 coronavirus useful for both diagnostic and research studies in South African institutions.

"I was meant to have been working on an influenza-surveillance project in 2020," Suliman told MRS Bulletin, "but, under the circumstances, I was required to apply my coronavirus expertise to the current pandemic. Thankfully, our funders were accommodating and we had enough resources among the collaborating research groups to be able to execute our COVID-19 research, but the funding implications for the future cannot be determined."

Furthermore, Suliman says, "There has been an incredible willingness between institutions to assist one another rather than compete, which saves a significant amount of time and resources." Suliman pointed out a collaboration with the University of Cape Town Lung Institute on various clinical projects that require her university's participation specifically in virus culture. In addition, their virus cultures have been of benefit to the SANational Health Laboratory Services and the University of the Witwatersrand, Johannesburg, for diagnostic and research purposes. 
Suliman says she could isolate the virus due to training she received in the laboratory of Christian Drosten, who now directs the Institute of Virology at the Charité University Hospital in Berlin. She now trains other researchers on the culturing of BSL-3 viruses.

\section{A call for relief packages}

In early April, the US House Science, Space, and Technology (SST) Committee actively sought input from the research community for ideas on funding and policy for R\&D and innovation that they would like to see in future stimulus packages. The Committee requested ideas directly related to COVID-19 R\&D, but also to help cover the impact of the pandemic on current research grants and facilities operation, and to cover costs and status for research students and fellows.

A number of "Dear Colleague" letters have also been issued by members of Congress seeking public endorsement of their request to government leadership to include the needs of research laboratories in upcoming stimulus packages. "Given the current shutdown of many universitybased laboratories and national laboratories due to the pandemic, we are deeply concerned that the people who comprise the backbone of the US research enterprise - graduate students, postdocs, principal investigators, and technical support staff - are at risk of losing their employment and their work," write Representatives Diana DeGette and Fred Upton. DeGette is a Democrat from the state of Colorado and Upton is a Republican from the state of Michigan. On April 29, they led over 180 lawmakers in requesting USD\$2 6 billion to be included in the next coronavirus stimulus package for scientific and medical researchers.

Back in March, a group of organizations, including the Association of American Universities, sent a letter to the congressional leadership in support of supplemental funding. Their main concern, due to laboratory shutdown, was the economic impact along with their ability to meet the requirements of their government grants and contracts.

Damon Dozier, Director of Government Affairs for the Materials Research
Society, told MRS Bulletin that the Energy Sciences Coalition responded to SST by reiterating an earlier request for government funding toward the infrastructure of DOE research facilities. "While the [DOE] Office of Science has been an excellent steward of these world-class scientific facilities," the coalition writes, "funding constraints have kept these facilities from undergoing the modernization necessary to remain state of the art." The coalition furthermore calls for funding to construct new world-class facilities and instruments. Specifically, they recommended:

- new and upgraded instruments at DOE Office of Science user facilities and shared research facilities, including those that build on the success of the Human Genome Project between DOE and the NIH to catalyze new collaborations in neuroscience and precision medicine;

- high-performance computing and networking infrastructure leveraging new Artificial Intelligence and Machine Learning (AI/ML) applications; and

- multidisciplinary quantum science and technology centers, quantum foundries, fellowships, and early-career research programs.

The Materials Research Society as well as sister societies including the American Chemical Society, the American Institute of Physics, and the American Physical Society are among the members of the coalition.

The Heroes Act, which passed a House vote on May 15, calls for USD\$4.745 billion to help the NIH expand COVID19-related research. Some of this funding is to go to "support the shutdown and startup costs of biomedical research laboratories nationwide" due to the pandemic, but no such stipulation has been made to other government $R \& D$ agencies or departments.

Ken Haenen, chair of the Department of Physics and director of the Doctoral School of Sciences and Technology at Hasselt University, Belgium, told MRS Bulletin that his research group has not benefited from the EU call for funding for COVID-19-related work. "Calls were very specific, and geared toward research directly linked to COVID-19, and my research has no link to that," says
Haenen, who researches carbon materials for energy harvesting and conversion, with a specific focus on chemical vapor deposition synthetic diamond. And there is "no extra budget foreseen" for research that has been delayed due to the pandemic.

However, Haenen says, project deadlines can be shifted and available funds can be extended to a longer time scale than originally planned. According to Haenen, each country in the EU has its own strategy in response to the pandemic. Similar to the EU calls for proposals, the government in Belgium - and even individual universities-have issued calls for research projects related to the current crisis. In Belgium, even though universities did not formally close, researchers turned to offcampus work for a few months. "My own university only now starts to chart possible problems that $\mathrm{PhD}$ researchers might have encountered due to corona-related delays, with special focus on people in the end phase of their PhD," Haenen says. The university response, he says, has not been "slow," but it is only now becoming clear what impact the pandemic has on research.

At the University of Cambridge, laboratory operations have been closed since March 30, Owens says, turning her research team to write articles based on their experiments. When the laboratories open, she expects that they will begin at $25 \%$ capacity. Any relief funding would come from the national government, she says.

Haenen does not expect a direct relief package at the EU or national levels, but a general concern is whether funding earmarked for COVID-19 R\&D is "extra." He says, "I think everybody understands that research is needed in this field, but if it siphons money away from other topics, there is always the danger that certain labs will suffer."

Prime Minister Justin Trudeau of Canada, in mid-May, acknowledged the many researchers who have turned their attention to COVID-19, leaving them to temporarily suspend their work from cancer treatments to clean technologies that protect the environment. In order to safeguard their research and enable them to continue their work after this crisis, Trudeau announced C\$450 million ( USD\$331 million) in funding. This is 
part of the country's COVID-19 Economic Response Plan. The funding will contribute toward salaries lost during suspension of research, support activities such as the safe storage of dangerous substances, and toward restarting data sets that were interrupted during the pandemic. Minister of Innovation, Science and Industry Navdeep Bains says, "By providing these institutions with immediate support, we continue to nurture Canada's talent pipeline and sustain our innovation capacity, while helping to mitigate the negative impacts of COVID-19 on the research community."

\section{Where to go from here}

Suliman would like to see a dedicated institute of virology in South Africa, with both commercial and academic involvement, where virus skills can be trained. "Unless we expand our virus culture capacity," Suliman says, "we will never be the ones who produce a new drug or vaccine, or who unlock the functions of new viral genes, or who find weak points in the virus that can be used as a vaccine or drug target, either for this or other viruses." South Africa has the researchers "to be able to compete internationally during this pandemic," she says, but lacks the opportunity and environment.

When asked about additional funding packages, Meng says, "I hope the federal government will launch concerted efforts

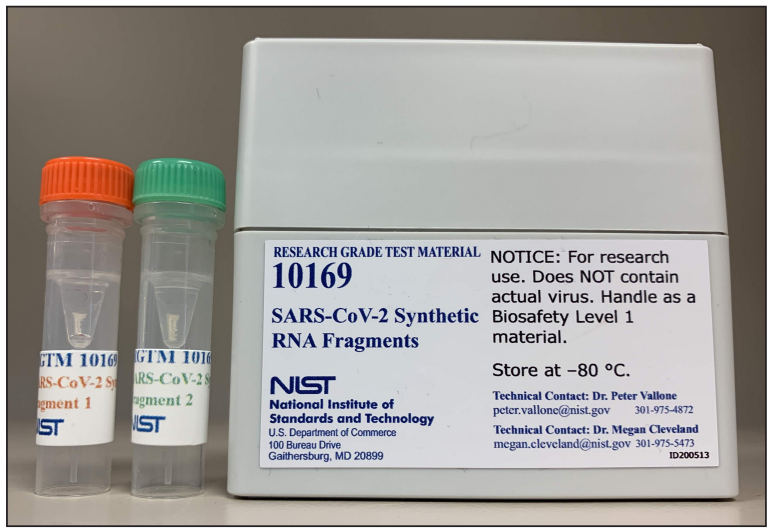

National Institute of Standards and Technology (NIST) SARS-CoV-2 Research Grade Test Material is a reference material of synthetic fragments of the SARS-CoV-2 virus RNA, which is the target of diagnostic tests for COVID-19. This material is intended to aid in evaluation of existing RT-qPCR assays and the development of new RT-qPCR assays, to be used as a calibrant in qPCR (dilutions), and to benchmark/compare other SARS-CoV-2 controls/materials. Credit: Megan Cleveland, NIST. to engage the scientific community, so we know how to better handle future challenges related to COVID." Rogers advocates for "funding for commercial translation of successful technology and research development efforts," and Ray says, "longterm funding for related research and education will be very helpful" to overcome the current and future pandemic situation.

Looking beyond the current pandemic or even a relief package to help laboratories that have been shut down, Hersam has another message for Capitol Hill. "I hope that funding for research related to combating future pandemics and other major problems that are likely to impact society can be provided in a proactive manner and sustained in the long term," he says. While appreciative of the immediate funds made available to fight the pandemic, Hersam says, "My strong belief is that the cost of prevention will inevitably be cheaper than reacting to a problem after it becomes an emergency."

In the case of COVID-19, Sapochak says it takes a confluence of several disciplines - engineering, physics, chemistry, biology, and math - to overcome the pandemic. "Materials research," she says, "embodies the very essence of convergence research." Researchers who work in the materials science realm know that by broadening perspective in this way, she says, they can solve problems more holistically and faster.

"As I look to the future," Sapochak says, "I see NSF continuing to be that resource that can facilitate and encourage this kind of convergence that expedites problem solving in novel ways."

Taking "holistic problem solving" to a different level, NSF - due to its emphasis on "broader impact"-is also positioned to fund studies about the effect of crises on different segments of the science, technology, engineering, and mathematics (STEM) community, with a particular interest in underrepresented groups. Sapochak told MRS Bulletin about an NSF Rapid Grant awarded by her division to Tabbetha Dobbins, Principal Investigator (Rowan University) and Thomas Searles, Co-principal Investigator (Howard University) on understanding the effects of COVID-19 campus closures on African American students in physics at the undergraduate level. Dobbins served on the American Institute of Physics Team-Up Task Force that issued a report on a two-year study about the persistent underrepresentation of African American students in physics. Based on their report, Dobbins is now directing this new study on "how support structures (e.g., mentoring, tutoring, intrusive advising) can be effectively implemented when students undergo crisis," using the pandemic as an example.

Dobbins and Searle write in their proposal that crises students face are often more personal, and that their study "bridges the learning from a sudden and collective crisis (i.e., COVID-19 campus closures) to well-designed actions that physics departments can take to address crises of a personal nature." The goal is to provide the appropriate support needed to help the students complete their degree and enter the workforce in their field of study. By funding this type of study, NSF ultimately contributes to diversifying the STEM field.

In South Africa, while preparations are in place for self-reliance, Nzimande simultaneously emphasized the importance of international cooperation where he embraced the UNESCO vision of open access, open data, and a science open to society. Nzimande had participated in the UNESCO virtual ministerial meeting on cooperation and open science in the fight against the pandemic that was held on March 30. UNESCO defines "open science" as "a movement aiming to make science more open, accessible, efficient, democratic, and transparent."

Examples of how such openness succeeds can be gleaned from the current pandemic situation. To locally build up a supply of PPE, South Africa - similar to other countries - turned its attention to 3D printers. In Stellenbosch University, the $3 \mathrm{D}$ printing laboratory that assisted 
surgeons in planning and rehearsing surgical procedures has now been redirected for PPE fabrication.

"There is a burgeoning community of people all over the world using 3D printers and laser cutters to produce equipment for healthcare workers, sharing designs and refining them, all being shared freely online," said Rudolph Venter, an orthopedic surgeon and lecturer at the university, according to a DSI news release. All of the materials needed are supplied locally.

In response to the pandemic, various departments within the University of Cambridge, in partnership with academics, engineers, intensive care medics, innovators, and industry partners across the UK and Africa, have formed a consortium for the Open Ventilator System Initiative (OVSI) for use in low- and middle-income countries. Current ventilators are expensive and difficult to fix, but an open-source design will allow users to adapt and fix the ventilators according to their needs and, by using readily available components, the machines can be built quickly across Africa in large numbers, according to a news release from the university.

Plastic injection molding is being used throughout the device to make the sophisticated machine simple and cheap to produce, according to OVSI. In response to a call for proposals from South Africa's Department of Trade, Industry and Competition, OVSI is working with two South African companies, Defy, a domestic appliance manufacturer, and Denel, a state-owned business, who will manufacture the ventilators locally. In the spring, Prodrive - a motorsport company in Oxfordshire, UK, developed a prototype, using a calibrated artificial lung for testing. The ventilator design will be released on an open-source license by the University of Cambridge.

In Italy, materials researcher Cristian Fracassi, who is the founder and CEO of the company Isinnova, received international news coverage for adapting a snorkeling mask into a hospital ventilator system. First the mask is converted into a CPAP (continuous positive airway pressure) mask. Fracassi's company then had a component designed called a Charlotte valve to connect the mask to the ventilator. The key to the Charlotte

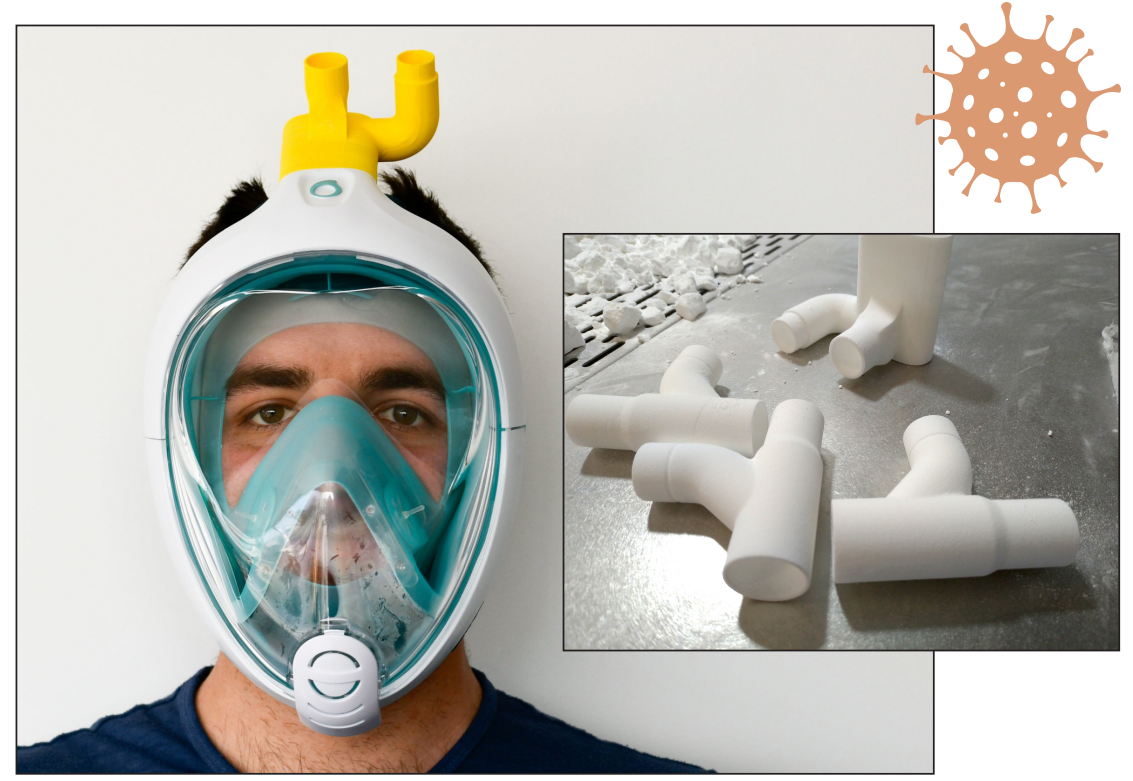

These 3D printed valves are produced by 3D Systems On Demand to convert a Decathlon snorkeling mask to an emergency ventilator mask. Designed by the company Issinova, located in northern Italy, the 3D printing instructions for the valve are available free. Full mask photo credit: Issinova. Inset credit: 3D Systems.

valve is that it can be easily $3 \mathrm{D}$-printed. The company recommends the use of PLA (polylactic) because it is odorless, biocompostable, and flexible.

According to Isinnova's news release, the company decided to "urgently patent the link valve" then render the patent free to use, "because it is in our intention that all hospitals in need could use it if necessary." The printing instructions are free, and the company is in the process of raising funds so that $3 \mathrm{D}$ printers, printing material, and technical support can be given free to those who need it. The company calls this effort "3D printing toward the future."

Similar to Singapore's claim of benefiting from previous $R \& D$ investment to contain the pandemic, South Africa points to its own R\&D investment in the Hydrogen South Africa (HySA) strategy initiated over 10 years ago. Together with the Department of Defence and Department of Public Works and Infrastructure, DSI is using a hydrogen fuel-cell system to provide clean power to temporary facilities - such as the Military Hospital in Pretoria- established as part of the government's response to COVID-19.

The modular nature of hydrogen fuel cells enables them to be moved from one place to another, giving them an important role in disaster management and distributed energy generation in remote areas. The HySA program relies on a platinumbased catalyst in a country that contains $75 \%$ of global platinum reserves. According to DSI, the production of renewable hydrogen from water requires significant quantities of platinum group metals. Local manufacturing of fuel cells and renewable hydrogen are therefore potential contributors to job creation and economic recovery post-COVID-19.

Looking ahead, Singapore's National Research Foundation issued a call for proposals in R\&D that will help society thrive in the "new COVID-19 normal." One theme called "Living with COVID19 " is looking for proposals regarding "personal health management, personal or community hygiene solutions, travel or commuting solutions ... and workplace solutions." The theme on "Rethinking Urban Designs" includes "sanitization systems in facilities and buildings."

In all, whether in containing the current pandemic, or preparing to live through future pandemics without repeating a heavy toll on life and a worldwide shutdown, R\&D in materials science is fundamental.

Judy Meiksin 